\title{
Sparstolonin B improves neurological outcomes following intracerebral hemorrhage in mice
}

\author{
YANCHUN WANG $^{1}$, SIDE JIANG $^{1}$, JING XIAO $^{1}$, QIAOLI LIANG ${ }^{2}$ and MINGSHAN TANG ${ }^{1}$ \\ ${ }^{1}$ Department of Neurology, Ba-Nan People's Hospital, Chongqing 401320; ${ }^{2}$ School of Pharmacy, Nanjing University of \\ Chinese Medicine, Jiangsu Collaborative Innovation Center of Chinese Medicinal Resources Industrialization, \\ Nanjing, Jiangsu 210023, P.R. China
}

Received August 2, 2017; Accepted February 2, 2018

DOI: $10.3892 /$ etm.2018.6092

\begin{abstract}
Inflammation serves an important role in inducing secondary injury following intracerebral hemorrhage (ICH). It has been demonstrated that sparstolonin B ( $\mathrm{SsnB})$ is able to attenuate the lipopolysaccharide-induced inflammatory response in sepsis. Mouse ICH models were used to explore the efficacy of $\mathrm{SsnB}$ on the ICH-induced inflammatory response. Mice underwent a working memory version of Morris water maze (MWM) test. They underwent 5 successive days of training consisting of 4 trials each day. The ICH model was established on the last training day. Mice were injected intraperitoneally either with vehicle or SsnB once a day for 3 consecutive days following the establishment of the ICH model. The MWM was used to determine the effect of SsnB on short-term memory following ICH. Neurological deficit scores and brain water content were measured following the MWM. Furthermore, the expression of inflammatory factors and signaling molecules downstream of TLR4 were measured. The results demonstrated that $5 \mathrm{mg} / \mathrm{kg}$ SsnB significantly improved the MWM path and time latency $(\mathrm{P}<0.05)$. Furthermore, neurological deficit scores were decreased in SsnB-treated mice compared with vehicle-treated mice $(\mathrm{P}<0.01)$. Brain water content, levels of inflammatory cytokines and the expression of inflammation-associated proteins were also significantly reduced in the $\mathrm{SsnB}$-treated group $(\mathrm{P}<0.05)$. These results indicate that $\mathrm{SsnB}$ treatment stimulates short-term neurobehavioral recovery and reduces neurological deficits and this may inhibit the inflammatory response. Therefore, SsnB may attenuate the inflammatory response following $\mathrm{ICH}$.
\end{abstract}

Correspondence to: Dr Mingshan Tang, Department of Neurology, Ba-Nan People's Hospital, 2 Xinnong Street, Chongqing 401320, P.R. China

E-mail: 1070363814@qq.com

Abbreviations: ELISA, enzyme-linked immunosorbent assay; $\mathrm{ICH}$, intracerebral hemorrhage; MWM, morris water maze; NDS, neurological deficit scores; SsnB, sparstolonin B; TLRs, toll-like receptors

Key words: intracerebral hemorrhage, toll-like receptors, inflammation, sparstolonin B

\section{Introduction}

Intracerebral hemorrhage (ICH) accounts for $10-15 \%$ of all strokes and is associated with high rates of mortality and morbidity $(1,2)$. Despite decades of research, the mortality rates of patients that have experienced $\mathrm{ICH}$ have scarcely improved (3). Primary brain injury caused by hematoma formation following ICH occurs, which causes mechanical damage to adjacent tissues. As hematoma is the most important factor for ICH outcome, early removal of the hematoma and prevention of hematoma expansion are potentially therapeutic $(4,5)$. However, the Surgical Trial in Intracerebral Hemorrhage (STICH) has failed to provide convincing evidence to support the efficacy of early surgical removal of hematoma (6). An increasing number of studies have focused on the mechanisms underlying ICH-induced secondary injury in order to identify novel therapeutic targets for ICH $(4,5)$. Several mechanisms contributing to secondary brain injury following ICH involve cytotoxic, excitotoxic, oxidative and inflammatory pathways $(5,7)$. It has been demonstrated that the inflammatory reaction that occurs following $\mathrm{ICH}$ serves a vital role in ICH-induced secondary brain injury.

Innate immunity and inflammatory responses are activated following ICH, potentially contributing towards the pathogenesis of secondary injury (8). Toll-like receptors (TLRs), which recognize distinct pathogen- and damage-associated molecular patterns, serve an important role in innate immunity and inflammatory responses $(9,10)$. It has been demonstrated that TLR4 is involved in the inflammatory response that occurs following ICH, which subsequently activates nuclear factor $(\mathrm{NF})-\kappa \mathrm{B}$ via the downstream myeloid differentiation primary response 88 (MyD88)/TIR-domain-containing adapter-inducing interferon- $\beta$ (TRIF) signaling pathway (11). Some natural compounds, including Curcumin (12) and Vitamin D (13), attenuated TLR4-induced inflammation injury in atherosclerosis and hyperoxia-induced lung injury. These results indicated that inhibiting the TLR4 signaling pathway may alleviate inflammation injury.

Previous studies have demonstrated that Sparstolonin B (SsnB), a natural compound extracted from the Chinese herb Scirpus yagara, may block the recruitment of MyD88 by TLR4 (14,15). MyD88 is critical for the TLR signaling pathway; inhibition of MyD88 results in the inhibition of the TLR signaling pathway and subsequently decreases 
inflammation (14). SsnB exhibits high liposolubility and has a low molecular weight, meaning that it can easily cross the blood-brain barrier (16). Therefore, SsnB may induce a therapeutic effect on the ICH-induced inflammatory response.

Therefore, the aim of the current study was to assess whether SsnB attenuates the inflammatory response following ICH. Autologous blood-induced mice ICH models were used to explore the efficacy of SsnB on ICH neurological outcomes in the present study. The Morris water maze (MWM) was used to observe the efficacy of SsnB on short memory following ICH. The neurological deficit scores (NDS), brain water content and the expression of inflammation-associated proteins were measured following the MWM testing.

\section{Materials and methods}

Animals. A total of 90 male C57BL/6 mice (8-10 weeks old, weighing 20-23 g) were obtained from the Animal Center of the Third Military Medical University (Chongqing, China). Animals were housed in individual cages in a specific pathogen-free facility $\left(25^{\circ} \mathrm{C}, 50-60 \%\right.$ humidity and a $12-12 \mathrm{~h}$ light-dark cycle) and had ad libitum access to sterile acidified water and radiation-treated food. The present study was approved by the Animal Ethics Committee of Ba-Nan People's Hospital (Chongqing, China) and were also conducted in accordance with the ARRIVE guidelines (17). Mice were randomly divided into the SsnB-treated group (45 mice) and the vehicle-treated group (45 mice) in the next experiment. No mice succumbed during the experiments.

Morris water maze (MWM). The MWM test was performed to assess spatial memory, as previously described $(18,19)$. The test requires the mice to swim to find a hidden transparent platform (10 $\mathrm{cm}$ in diameter; submerged $1 \mathrm{~cm}$ below the surface of the water) and to use distal spatial cues and their configuration to remember its location. The mice could not see the submerged platform as the water was made opaque using non-toxic paint (Primalex; PPG Deco, Břasy, Czech Republic). The MWM pool, had a diameter of $160 \mathrm{~cm}$ and was filled up with water up to $40 \mathrm{~cm}$. The pool was placed in a room with an abundance of visual landmarks and water temperature was maintained at $20^{\circ} \mathrm{C}$.

In the training phase, mice underwent 4 trials/day over 5 consecutive days. Each mouse was given $60 \mathrm{sec}$ to find the platform. If the mice found the platform in $<60 \mathrm{sec}$, the mice were allowed to rest on the platform for $30 \mathrm{sec}$. If the mice could not find the platform in the $60 \mathrm{sec}$ time period, it was gently guided towards it by the experimenter and subsequently allowed to rest for $30 \mathrm{sec}$ to emphasize the platform.

In the testing phase, a computerized video tracking system (Supermaze Morris; Shanghai Xinruan Information Technology Co., Ltd., Shanghai, China) recorded the path latency from entering the water until the platform was found and calculated the swimming time. The first 5 days were the training phase prior to the establishment of $\mathrm{ICH}$ models on day 5. The actual trials were conducted over 3 successive days on day 2 following the establishment of the ICH model. Mice were released in the quadrant diagonally opposite to the location of the previous platform and allowed to swim for $60 \mathrm{sec}$. If mice found the platform in $<60 \mathrm{sec}$, the time spent in the water and path latency were recorded as the actual time and path latency. If the mice could not find the platform after $60 \mathrm{sec}$ time, the time spent was recorded as $60 \mathrm{sec}$, and the actual path latency was recorded for $60 \mathrm{sec}$.

ICH model. The ICH model was established in all mice following a previously reported protocol (20). All animals were anesthetized with $4 \%$ chloral hydrate at a dose of $400 \mathrm{mg} / \mathrm{kg}$ by intrapenitoneal injection and immobilized in a stereotaxic frame (Stoelting Co., Wood Dale, IL, USA). Autologous whole blood (25 $\mu 1$, without anticoagulant, drawn from the tip of the tail) was injected into the striatum $(0.2 \mathrm{~mm}$ anterior and $2.3 \mathrm{~mm}$ lateral to the bregma, $3.5 \mathrm{~mm}$ deep from the skull) of each mouse using a syringe pump (Lagato100, KD Scientific, Inc., Holliston, MA, USA). Each mouse initially received a $5 \mu \mathrm{l}$ injection of whole blood and after $7 \mathrm{~min}$, received a second injection of blood $(20 \mu \mathrm{l})$ at $2 \mu \mathrm{l} / \mathrm{min}$.

SsnB treatment. SsnB was extracted and prepared from the rhizome of the Chinese herb Scirpus yagara (Kangmei Century Chinese Medicine Co. Ltd., Bozhou, China) as described previously $(14,15)$. Scirpus yagara was widely used in traditional Chinese medicine (14). A previous study revealed that SsnB reduced lipopolysaccharide (LPS)-induced nuclear factor (NF)- $\kappa \mathrm{B}$ activity in a dose-dependent manner and indicated that the half-maximal inhibitory concentration of SsnB is $\sim 5 \mathrm{mg} / \mathrm{kg} /$ day (14). Therefore, $5 \mathrm{mg} / \mathrm{kg} \mathrm{SsnB}$, dissolved in polyethylene glycol 400 (cat. no. 1546445; Sigma Aldrich, Merck KGaA, Darmstadt, Germany), was injected intraperitoneally into 45 mice $2 \mathrm{~h}$ after the establishment of the ICH model and subsequently once a day at the same time everyday over 3 consecutive days. The mice in the control group (45 mice) were injected intraperitoneally with the same volume $(0.1 \mathrm{ml})$ of vehicle (polyethylene glycol 400) $2 \mathrm{~h}$ after the establishment of the ICH model and subsequently once a day at the same time everyday over 3 consecutive days.

A total of 40 mice (20 mice/group) were monitored by two blinded observers during the MWM and neurological deficit score (NDS) tests. Following these tests the 40 mice were sacrificed. A total of 20 were sacrificed 1 day after the ICH model was established (10 mice/group) and 20 were sacrificed 3 days after the ICH model was established (10 mice/group).

The remaining 50 mice ( 25 mice/group) were sacrificed 1 day (a total of 20 mice, 10 mice/group) and 3 days (a total of 30 mice, 15 mice/group) after ICH was established. These mice were sacrificed for brain water content, ELISA or western blot analysis.

NDS. Different behavioral tests, including postural flexing, forelimb placing, circling and foot fault tests, were used to assess neurological deficits, as reported previously $(21,22)$. Each test was scored from 0-4, with a maximum possible deficit score of 16. Two trained investigators who were blinded to animal grouping scored the tests and the mean score of the subscales was the final score for each mouse.

Determination of brain water content. Brain water content was measured following a previously described protocol $(20,23)$. Brains were removed and 3-mm coronal slices were obtained. Each brain slice was divided into two hemispheres along the midline and for each hemisphere, the cortex and basal ganglia 
were dissected. Wet and dry weights were recorded for each section prior to and following incubation of the sections at $95-100^{\circ} \mathrm{C}$ for $24 \mathrm{~h}$, respectively. The brain water content was calculated using the following formula: (Wet weight-dry weight)/wet weight $\times 100 \%$.

Enzyme-linked immunosorbent assay (ELISA). Perihematomal brain tissue samples from 10 mice ( $\mathrm{n}=5 /$ group) were centrifuged $\left(4^{\circ} \mathrm{C}\right.$ at $12,000 \times$ g for $\left.15 \mathrm{~min}\right)$ and the concentrations of tumor necrosis factor (TNF)- $\alpha$ (mouse TNF- $\alpha$ ELISA kit; cat. no., DKW12-2720-096), interleukin (IL)-1 $\beta$ (mouse IL-1 $\beta$ ELISA kit; cat. no., DKW12-1012-096) and IL-6 (mouse IL-6 kit; cat. no., DKW12-1060-096) in the supernatant were measured using ELISA kits following the manufacturer's protocol (Dakewe, Shenzhen, China).

Western blotting. A total of 10 mice ( $\mathrm{n}=5 /$ group) were perfused with $0.01 \mathrm{~mol} / 1$ PBS 3 days after ICH, and the cerebral tissues from the perihematomal region were isolated $(n=5)$. The protein was extracted and the protein concentration was determined using the nuclear and cytoplasmic protein extraction kit (cat. no. P0027) and enhanced BCA protein assay kit (cat. no. P0010; Beyotime Institute of Biotechnology, Haimen, China), respectively, according to the manufacturer's protocol. A total of $50 \mu \mathrm{g}$ protein was loaded per lane by SDS-PAGE in a $8 \%$ separation gel and 5\% spacer gel. The proteins were then transferred onto polyvinylidene fluoride membranes (GE Healthcare, Chicago, IL, USA). Membranes were blocked using $0.5 \%$ goat serum (cat. no. C0265; Beyotime Institute of Biotechnology) at $25^{\circ} \mathrm{C}$ for $2 \mathrm{~h}$ and then incubated with rabbit anti-mouse TLR4 (1:200; cat. no. ab13556), rabbit anti-mouse TRIF (1:400; cat. no. ab13810), rabbit anti-mouse MyD88 (1:400; cat. no. ab135693; all Abcam, Cambridge, UK) or rabbit anti-mouse NF-kB (1:400; cat. no. AN365; Beyotime Institute of Biotechnology) antibodies at $4^{\circ} \mathrm{C}$ overnight. Rabbit monoclonal anti-mouse GAPDH antibodies (1:1,000; cat. no. AF1186; Beyotime Institute of Biotechnology) were used as a loading control. Subsequently, membranes were incubated with horseradish peroxidase-conjugated goat anti-rabbit secondary antibodies (1:3,000; cat. no. sc2004, Santa Cruz Biotechnology, Inc., Dallas, TX, USA) at $25^{\circ} \mathrm{C}$ for $1.5 \mathrm{~h}$. Immunoreactive bands were visualized using a chemiluminescence detection system (BeyoECL Star; Beyotime Institute of Biotechnology) in the chemiluminescence imager (FluorQuant ${ }^{\mathrm{TM}}$ AC600; Acuronbio Technology LLC, Houston, TX, USA). Densitometric analysis was performed using Image-Pro Plus 6.0 software (Media Cybernetics, Inc., Rockville, MD, USA).

Statistical analysis. All data are presented as the mean \pm standard deviation. Statistical analyses were performed using the statistical software SPSS version 13.0 (SPSS, Inc., Chicago, IL, USA). Levene's homogeneity of variances test was used to test the variance homogeneity. Student $t$ test was used to compare differences between two groups and $\mathrm{P}<0.05$ was considered to indicate a statistically significant difference.

\section{Results}

SsnB improves neurobehavioral performance following ICH. The coronal level (and volume) of mice following ICH is

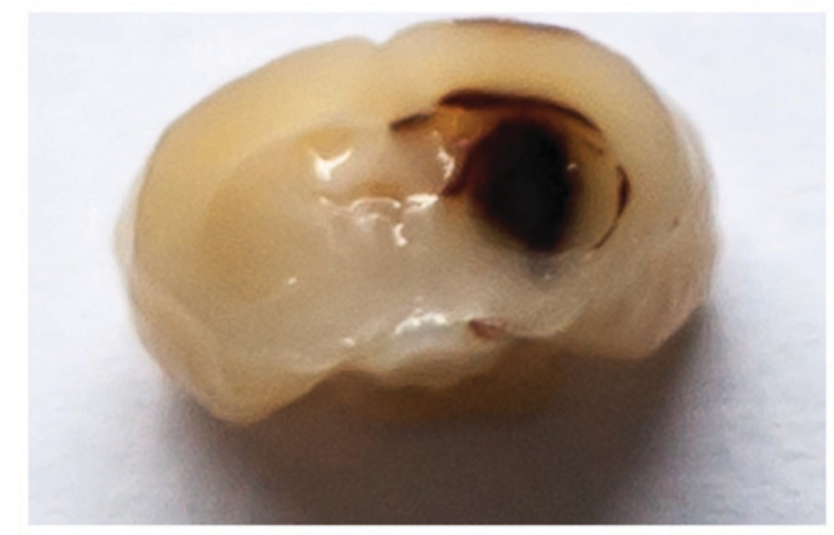

Figure 1. The coronal level (and volume) following intracerebral hemorrhage. The hematoma located at the caudate nucleus of the mice. The tissues used in ELISA and western blot analyses were collected from the perihematoma tissue.

presented in Fig. 1. The hematoma was located at the caudate nucleus of the mice. At the end of the training period, there were no differences in average swimming path latency and time latency between the two groups (Fig. 2A and B). To determine the effect of SsnB on spatial memory; time and path latency were measured 1 and 3 days following the establishment of ICH models. Mice in the ICH $+\mathrm{SsnB}$ group exhibited significantly lower latency compared with the ICH + vehicle group at each time point $(13.1 \pm 5.3$ vs. $23.4 \pm 9.9, \mathrm{P}=0.01 ; 7.8 \pm 5.4$ vs. $13.3 \pm 5.9, \mathrm{P}=0.04$, respectively; Fig. $2 \mathrm{C}$ ). Path latency in the $\mathrm{ICH}+\mathrm{SsnB}$ group was also significantly lower than that of the $\mathrm{ICH}+$ vehicle group 1 and 3 days following ICH establishment $(4.2 \pm 1.9$ vs. $7.3 \pm 3.1, \mathrm{P}=0.01 ; 2.8 \pm 1.4$ vs. $5.0 \pm 2.5, \mathrm{P}=0.03$ respectively, Fig. 2D).

To evaluate neurobehavioral performance between the two groups, NDS were measured. The NDS of the ICH + SsnB group were significantly lower than those of the ICH + vehicle group 1 and 3 days following the establishment of ICH models ( $8.5 \pm 2.1$ vs. $11.2 \pm 1.7, \mathrm{P}=0.003 ; 6.6 \pm 1.8$ vs. $9.1 \pm 1.6, \mathrm{P}=0.004$, respectively, Fig. 3A).

\section{$S s n B$ reduces brain edema and the expression of inflammatory} factors in mice following ICH. Brain water content and the expression of inflammatory factors were subsequently evaluated following euthanasia. The dry-wet weight method was used to determine the effect of SsnB on brain edema following ICH. The results indicated that SsnB significantly decreased brain water content in the ipsilateral cortex and basal ganglia of mice compared with the ICH + vehicle group 1 and 3 days following the induction of ICH $(\mathrm{P}<0.05$, Fig. 3B). These results suggest that $\mathrm{SsnB}$ significantly reduces brain edema in mice with ICH.

Inflammatory factors are critical indices for inflammatory reaction following ICH. ELISA was used to determine levels of pro-inflammatory factors, including TNF- $\alpha$, IL- $1 \beta$, and IL-6, in the perihematomal tissue of mice following ICH. Levels of TNF- $\alpha$, IL-1 $\beta$, and IL- 6 were all significantly decreased in the ICH + SsnB group compared with the $\mathrm{ICH}+$ vehicle group at 1 and 3 days following the establishment of $\mathrm{ICH}$ $(\mathrm{P}<0.05$; Fig. $3 \mathrm{C})$. These results indicate that $\mathrm{SsnB}$ reduces the production of inflammatory factors in perihematoma tissue in a mouse model of ICH. 
A

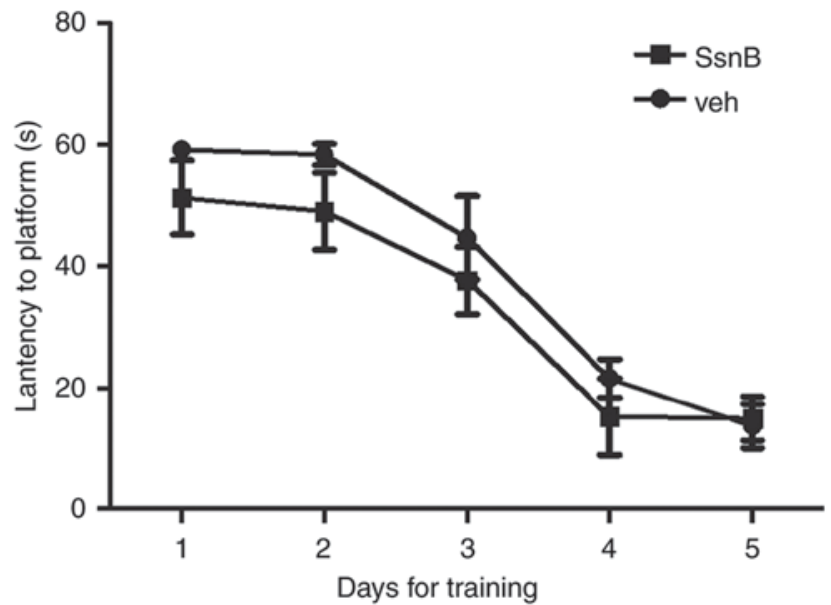

$\mathrm{B}$

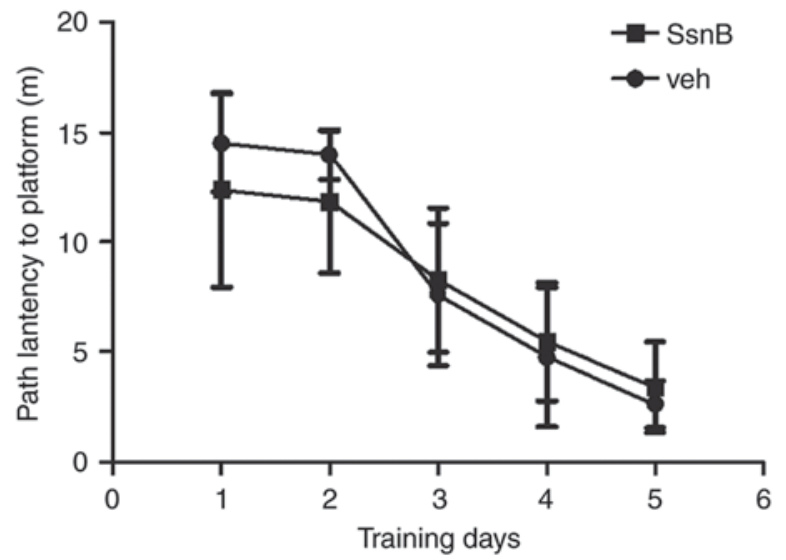

C
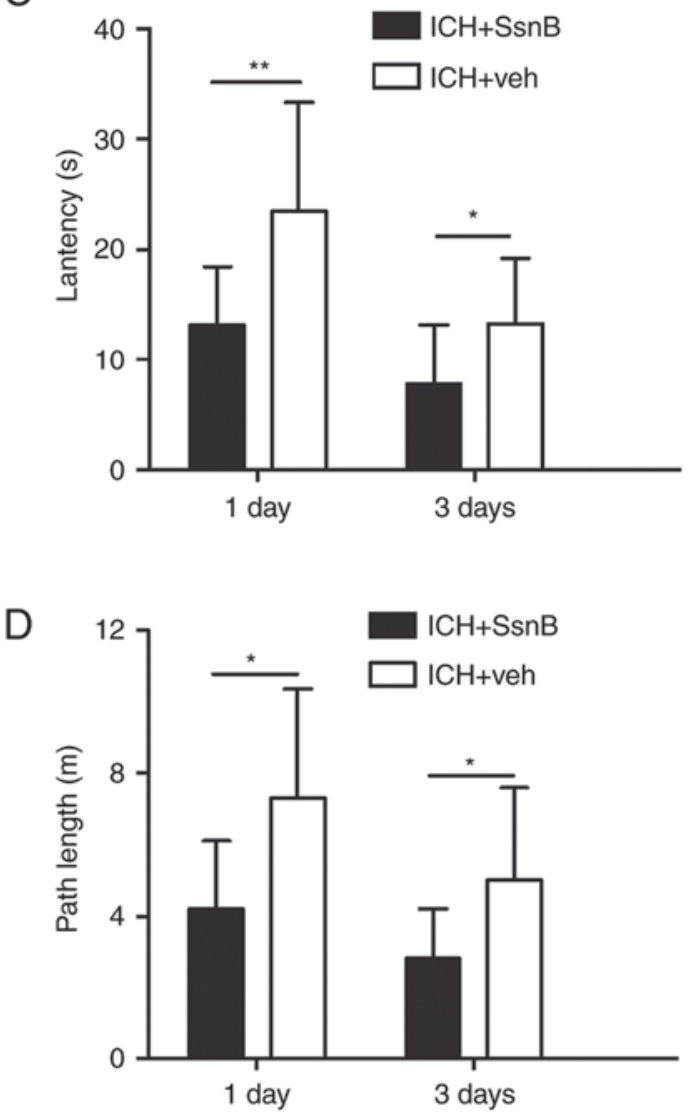

Figure 2. Effect of SsnB on the working memory of mice with ICH. The effect of training on the results of the Morris water maze test indicated the decreased (A) time and (B) path latencies of experimental mice over 5 consecutive trials. Following the establishment of ICH, one group of mice received SsnB treatment, which reduced the (C) latency to platform and the (D) path latency to platform on days 1 and 3 following the establishment of ICH. All data are presented as the mean \pm standard deviation. $(\mathrm{n}=10)^{*} \mathrm{P}<0.05$ and ${ }^{* *} \mathrm{P}<0.01$. ICH, intracerebral hemorrhage; Ssn B, Sparstolonin B.
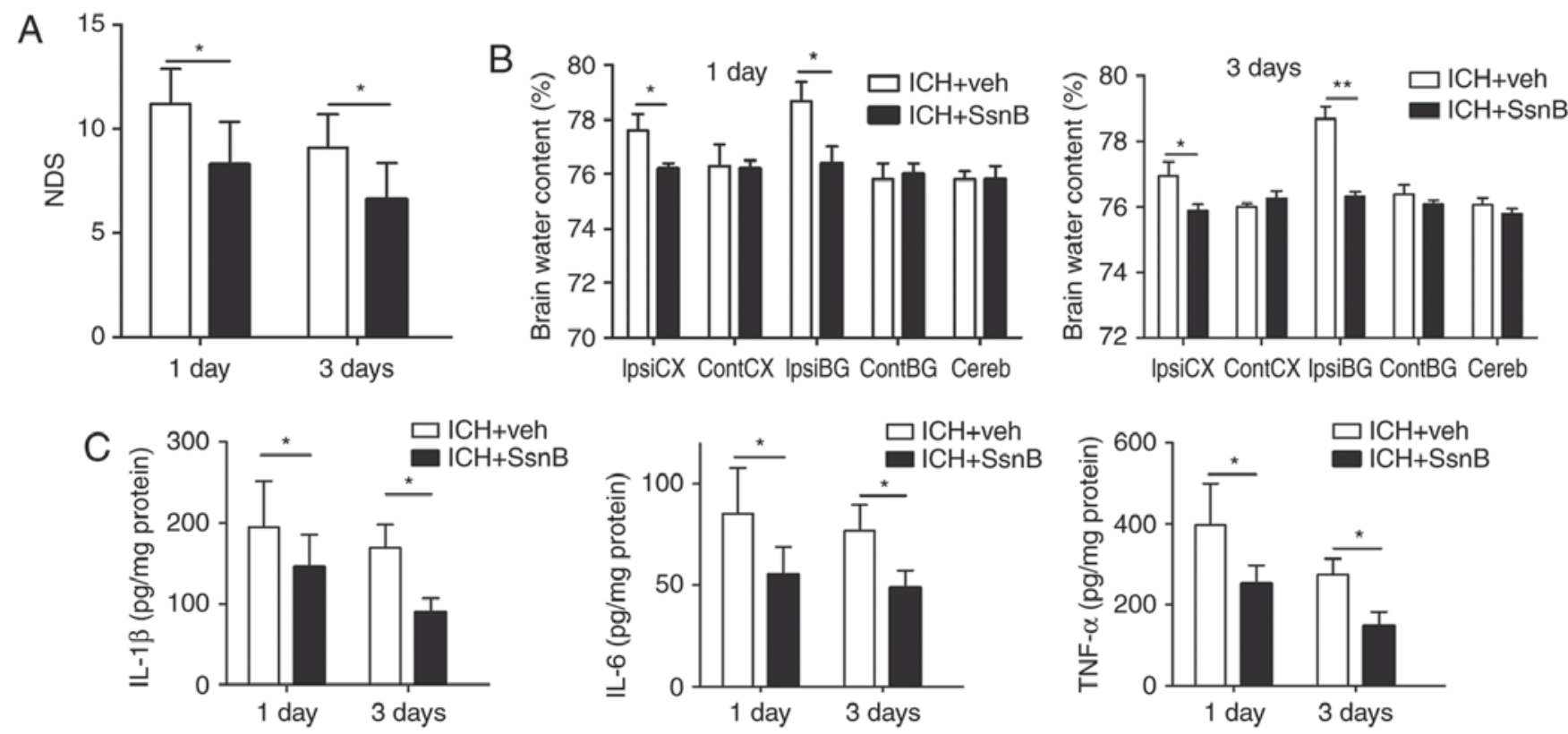

Figure 3. SsnB reduces the inflammatory response in autologous blood-induced ICH mouse models. (A) Intravenous injection of SsnB reduced NDS in autologous-induced ICH mice $(n=10)$. (B) Intravenous injection of SsnB reduced brain water content following ICH ( $\mathrm{n}=5)$. $(\mathrm{C}) \mathrm{The}$ concentrations of TNF- $\alpha$, IL-1 $\beta$, and IL-6 in perihematomal tissues in ICH mice $(n=5)$. All data are presented as the mean \pm standard deviation. Data were obtained for samples pooled from 5 mice, and the experiments were repeated 3 times. ${ }^{*} \mathrm{P}<0.05$ and ${ }^{* *} \mathrm{P}<0.01$. ContCX, contralateral cortex; ContBG, contralateral basal ganglia; IpsiCX, ipsilateral cortex; IpsiBG, ipsilateral basal ganglia; Cereb, cerebellum; ICH, intracerebral hemorrhage; SsnB, Sparstolonin B; IL, interleukin; TNF, tumor necrosis factor; NDS, neurological deficit scores. 
A
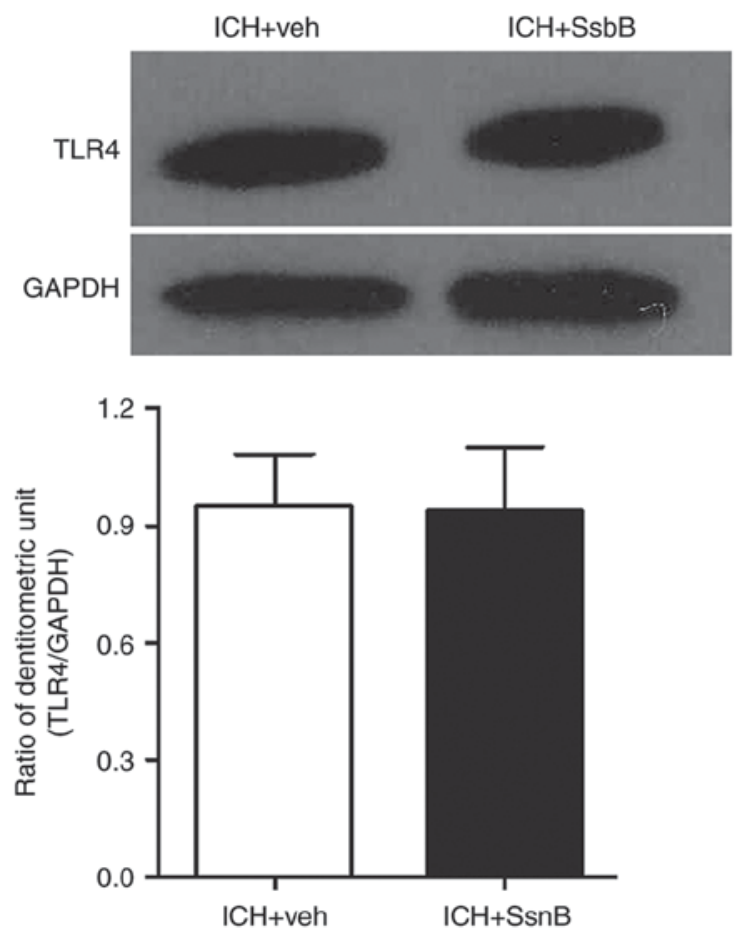

C
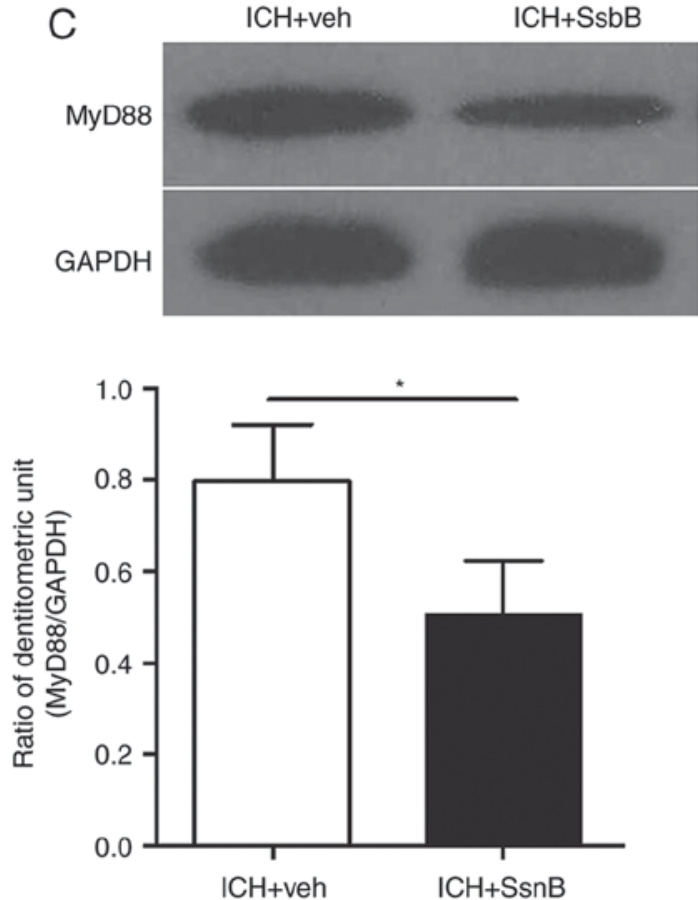

B

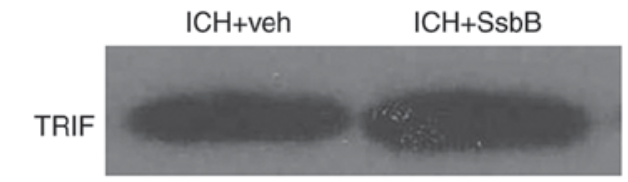

GAPDH

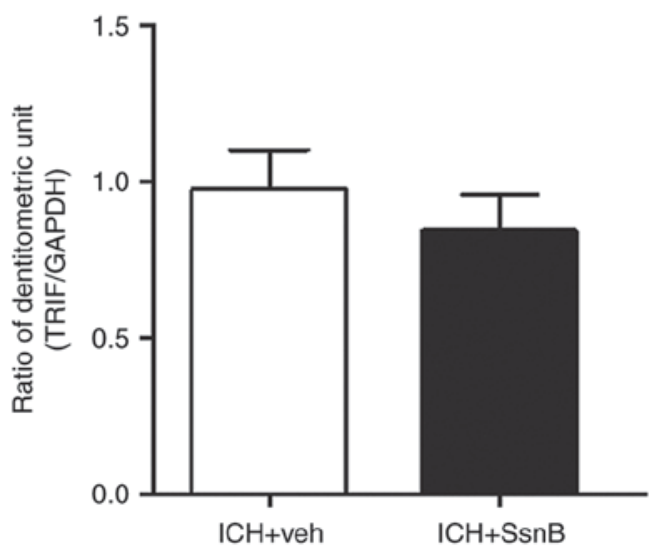

$\mathrm{D}$
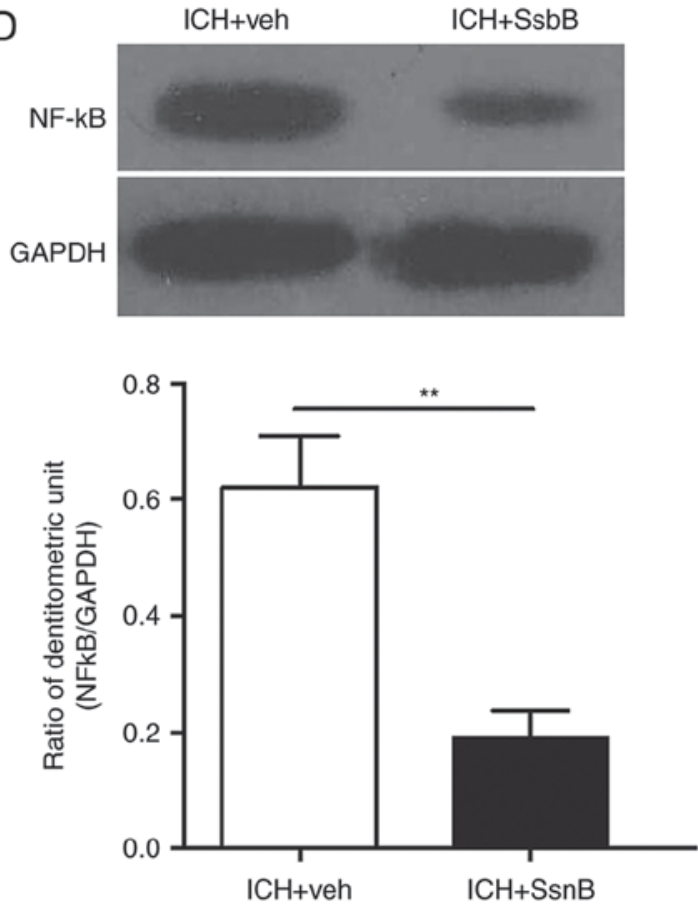

Figure 4. SsnB reduced the expression of MyD88 and NF- $\mathrm{kB}$ in perihematomal tissues following ICH. The expression of (A) TLR4 and (B) TRIF in perihematomal tissue following ICH did not differ between the ICH + vehicle and ICH $+\mathrm{SsnB}$ groups. Compared with the ICH + vehicle group, SsnB reduced (C) MyD88 and (D) NF-kB expression in perihematomal tissue following ICH. All data are presented as the mean \pm standard deviation. Data were obtained for samples pooled from 5 mice, and the experiments were repeated 3 times. ${ }^{*} \mathrm{P}<0.05$ and ${ }^{* *} \mathrm{P}<0.01, \mathrm{n}=5$. ICH, intracerebral hemorrhage; SsnB, Sparstolonin $\mathrm{B}$; TRIF, TIR-domain-containing adapter-inducing interferon- $\beta$; TLR4, toll-like receptor 4 ; NF- $\kappa \mathrm{B}$, nuclear factor $\kappa \mathrm{B}$; MyD88, myeloid differentiation primary response 88.

SsnB affects the expression of signaling molecules associated with TLR4. Previous studies indicated that SsnB suppressedTLR4-mediated inflammatory reaction in an LPS-induced sepsis mouse model $(14,15)$. To further evaluate the underlying neuroprotective mechanism of SsnB in ICH, western blotting was performed to examine the expression of signaling molecules downstream of TLR4.
The expression of TLR4, TRIF, MyD88 and NF- $\mathrm{kBp} 65$ in perihematomal tissues 3 days following ICH were measured. The results indicated that there were no differences in the expression of TLR4 and TRIF between mice in the $\mathrm{ICH}+$ vehicle group compared with those in the $\mathrm{ICH}+\mathrm{SsnB}$ group 3 days following ICH (Fig. 4A and B). However, the expression of MyD88 and NF- $\mathrm{kB}$ were significantly decreased 
in the perihematomal tissues of mice in the $\mathrm{ICH}+\mathrm{SsnB}$ group compared with the ICH + vehicle group 3 days following $\mathrm{ICH}$ $(\mathrm{P}<0.05$ and $\mathrm{P}<0.01$, respectively; Fig. 4C and $\mathrm{D})$.

\section{Discussion}

Inflammatory processes serve a critical role in the secondary damage following $\mathrm{ICH}$; indeed, several studies have indicated that the inflammatory reaction is one of the main mechanisms that induces the secondary damage associated with ICH $(24,25)$. Secondary brain injury following ICH includes hematoma toxicity, high metabolic injury, excitotoxicity, oxidative stress and inflammatory injury (1,9-11). Previous studies into injury following $\mathrm{ICH}$ have focused downstream of inflammation and have not identified reagents that induce sufficient therapeutic effects in patients with $\mathrm{ICH}(5,8)$.

Toll-like receptors (TLRs) serve a key role in innate immunity and inflammatory responses $(9,10)$. TLR4 signaling in inflammatory responses induces secondary injury following $\mathrm{ICH}$, which subsequently activates $\mathrm{NF}-\kappa \mathrm{B}$ via the downstream MyD88/TRIF signaling pathway (11). Furthermore, it has been demonstrated that TLR4 contributes to poor outcomes following intracerebral hemorrhage. Therefore, the aim of the current study was to suppress secondary injury following the initial inflammation reaction, thus alleviating $\mathrm{ICH}$-induced injury and reducing associated neurological deficits.

SsnB is a natural compound extracted from the Chinese herb, Scirpus yagara, which is a perennial, aquatic plant whose tubers have long been used in traditional Chinese medicine to treat several inflammatory diseases (14). It has been demonstrated that high concentrations of SsnB have no toxic effects on macrophages in rats with sepsis (15). Therefore, it was speculated that it may induce no side effect in the ICH model. The current study did not measure the side effects of SsnB; therefore, it remains unknown whether SsnB induces side effects in a mouse model of ICH and further studies are required. SsnB has a low molecular weight and high lipid solubility, which facilitates its ability to cross the blood brain barrier and target an ICH-induced inflammatory response $(15,16)$. Therefore $\mathrm{SsnB}$ may be developed as a potential method of treating patients with ICH.

It has been reported that $\mathrm{SsnB}$ inhibits the production of multiple cytokines and protects mice against endotoxin shock (26). Liu et al (27) demonstrated that SsnB attenuates hypoxia-reoxygenation-induced cardiomyocyte inflammation by inhibiting the TLR2/4-mediated inflammatory response. The results of the present study may provide valuable insights into the mechanisms and potential treatments of other bleeding diseases, including gastrointestinal bleeding and Crush syndrome.

The results of the current study demonstrated that SsnB suppresses the expression of MyD88 and NF- $\kappa$ B p65, which ultimately induces the downregulation of pro-inflammatory factors, including IL-1 $\beta$, IL-6 and TNF- $\alpha$. SsnB did not affect the expression of TLR4 and TRIF. This indicates that SsnB does not affect TLR4 expression or TLR4-TRIF signaling, which induces the expression of interferon regulatory factor 3 (IRF-3). Consecutively, activation of the IRF-3 signaling pathway induces the expression of interferon, which is an antiviral, antitumor and immune regulatory factor (28). The results of the current study indicate that the underlying neuroprotective mechanism of SsnB may be associated with MyD88 and are consistent with the results of a study by Zhong et al (16), which indicated that SsnB may inhibit TLR2/TLR4 heterodimer formation on cultured bone marrow-derived dendritic cells and that this mechanism was associated with MyD88 Arg196. The current study did not examine SsnB concentrations in perihematomal tissues following ICH. Therefore, further studies are required to determine the plasma concentration and pharmacokinetics of SsnB in mice following ICH.

There were no differences between the time and path latencies in the two groups on days 4 and 5 of the MWM training. This indicates that swimming path and latency tend to be stable. Hence, ICH models were established on day 5 following the training experiment.

In the mouse models of $\mathrm{ICH}$, the time and path latencies of mice in the SsnB treated group were significantly lower compared with the vehicle group. These results indicate that SsnB may improve short-term spatial memory following $\mathrm{ICH}$; however, the effect of SsnB on long-term spatial memory was not tested in the current study. It remains unknown whether SsnB may improve short-term memory in other neurological diseases.

In conclusion, SsnB significantly improved the MWM path and time latency. The neurological deficit scores were decreased in SsnB-treated mice. Brain water content, the levels of inflammatory cytokines and the expression of inflammation-associated proteins were also significantly reduced in the SsnB-treated group. These results indicate that SsnB treatment stimulates short-term neurobehavioral recovery and reduces neurological deficits. SsnB may attenuate the ICH-induced inflammatory response and may be developed as a natural therapeutic candidate for ICH treatment.

\section{Acknowledgments}

Not applicable.

\section{Funding}

The present study was supported by grants from the National Natural Science Foundation of China (grant no. 81400974).

\section{Availability of data and materials}

All data generated or analyzed during this study are included in this published article.

\section{Authors' contributions}

YW conceived of and coordinated the study, and designed, performed and analyzed the experiments. SJ and JX performed the data analysis and collected the data. YW wrote the paper. QL and MT designed and revised the paper. All authors reviewed the results and approved the final version of the manuscript.

\section{Ethics approval and consent to participate}

The present study was approved by the Animal Ethics Committee of Ba-Nan People's Hospital (Chongqing, China) 


\section{Consent for publication}

Not applicable.

\section{Competing interests}

The authors declare that they have no conflicts of interest.

\section{References}

1. Qureshi AI, Mendelow AD and Hanley DF: Intracerebral haemorrhage. Lancet 373: 1632-1644, 2009.

2. van Asch CJ, Luitse MJ, Rinkel GJ, van der Tweel I, Algra A and Klijn CJ: Incidence, case fatality, and functional outcome of intracerebral haemorrhage over time, according to age, sex and ethnic origin: A systematic review and meta-analysis. Lancet Neurol 9: 167-176, 2010.

3. Zahuranec DB, Lisabeth LD, Sánchez BN, Smith MA, Brown DL, Garcia NM, Skolarus LE, Meurer WJ, Burke JF, Adelman EE and Morgenstern LB: Intracerebral hemorrhage mortality is not changing despite declining incidence. Neurology 82: 2180-2186, 2014.

4. Xi G, Strahle J, Hua Y and Keep RF: Progress in translational research on intracerebral hemorrhage: Is there an end in sight? Prog Neurobiol 115: 45-63, 2014.

5. Zhou Y, Wang Y, Wang J, Anne Stetler R and Yang QW: Inflammation in intracerebral hemorrhage: From mechanisms to clinical translation. Prog Neurobiol 115: 25-44, 2014.

6. Mendelow AD, Gregson BA, Fernandes HM, Murray GD Teasdale GM, Hope DT, Karimi A, Shaw MD and Barer DH; STICH investigators: Early surgery versus initial conservative treatment in patients with spontaneous supratentorial intracerebral haematomas in the International Surgical Trial in Intracerebral Haemorrhage (STICH): A randomised trial. Lancet 365: 387-397, 2005 .

7. Aronowski J and Zhao X: Molecular pathophysiology of cerebral hemorrhage: Secondary brain injury. Stroke 42: 1781-1786, 2011.

8. Wang J: Preclinical and clinical research on inflammation after intracerebral hemorrhage. Prog Neurobiol 92: 463-477, 2010.

9. Akira S, Uematsu S and Takeuchi O: Pathogen recognition and innate immunity. Cell 124: 783-801, 2006.

10. Kong Y and Le Y: Toll-like receptors in inflammation of the central nervous system. Int Immunopharmacol 11: 1407-1414, 2011.

11. Wang YC, Zhou Y, Fang H, Lin S, Wang PF, Xiong RP, Chen J, Xiong XY, Lv FL, Liang QL and Yang QW: Toll-like receptor $2 / 4$ heterodimer mediates inflammatory injury in intracerebral hemorrhage. Ann Neurol 75: 876-889, 2014.

12. Zhang S, Zou J, Li P, Zheng X and Feng D: Curcumin protects against atherosclerosis in apolipoprotein E-knockout mice by inhibiting toll-like receptor 4. expression. J Agr Food Chem 66: 449-456, 2018.

13. Yao L, Shi Y, Zhao X, Hou A, Xing Y, Fu J and Xue X: Vitamin $\mathrm{D}$ attenuates hyperoxia-induced lung injury through downregulation of Toll-like receptor 4. Int J Mol Med 39: 1403-1408, 2017.
14. Liang Q, Wu Q, Jiang J, Duan J, Wang C, Smith MD, Lu H, Wang Q, Nagarkatti P and Fan D: Characterization of sparstolonin B, a Chinese herb-derived compound, as a selective Toll-like receptor antagonist with potent anti-inflammatory properties. J Biol Chem 286: 26470-26479, 2011.

15. Liang QL, Lei LL, Cui X, Zou NS and Duan JA: Bioactive cis-stilbenoids from the tubers of Scirpus yagara. Fitoterapia 84: 170-173, 2013.

16. Zhong Q, Zhou K, Liang QL, Lin S, Wang YC, Xiong XY, Meng ZY, Zhao T, Zhu WY, Yang YR, et al: Interleukin-23 secreted by activated macrophages drives $\gamma \delta \mathrm{T}$ cell production of interleukin-17 to aggravate secondary injury after intracerebral hemorrhage. J Am Heart Assoc 5: e004340, 2016.

17. Kilkenny C, Browne WJ, Cuthill IC, Emerson M and Altman DG: Improving bioscience research reporting: The ARRIVE guidelines for reporting animal research. PLoS Biol 8: e1000412, 2010.

18. Vorhees CV and Williams MT: Value of water mazes for assessing spatial and egocentric learning and memory in rodent basic research and regulatory studies. Neurotoxicol Teratol 45: 75-90, 2014

19. Terry AV Jr: Spatial navigation (Water Maze) tasks. In: Methods of behavior analysis in neuroscience. 2nd edition. Buccafusco JJ (ed). Boca Raton (FL), 2009.

20. Wang YC, Wang PF, Fang H, Chen J, Xiong XY and Yang QW: Toll-like receptor 4 antagonist attenuates intracerebral hemorrhage-induced brain injury. Stroke 44: 2545-2552, 2013.

21. Zhao X, Zhang Y, Strong R, Grotta JC and Aronowski J: $15 \mathrm{~d}$-Prostaglandin J2 activates peroxisome proliferator-activated receptor-gamma, promotes expression of catalase, and reduces inflammation, behavioral dysfunction, and neuronal loss after intracerebral hemorrhage in rats. J Cereb Blood Flow Metab 26 811-820, 2006

22. Lin S, Yin Q, Zhong Q, Lv FL, Zhou Y, Li JQ, Wang JZ, Su BY and Yang QW: Heme activates TLR4-mediated inflammatory injury via MyD88/TRIF signaling pathway in intracerebral hemorrhage. J Neuroinflamm 9: 46, 2012.

23. Chang CF, Chen SF, Lee TS, Lee HF and Shyue SK: Caveolindeletion reduces early brain injury after experimental intracerebral hemorrhage. Am J Pathol 178: 1749-1761, 2011.

24. Belur PK, Chang JJ, He S, Emanuel BA and Mack WJ: Emerging experimental therapies for intracerebral hemorrhage: Targeting mechanisms of secondary brain injury. Neurosurg Focus 34: E9, 2013.

25. Ziai WC: Hematology and inflammatory signaling of intracerebral hemorrhage. Stroke 44 (6 Suppl 1): S74-S78, 2013.

26. Liang Q, Dong S, Lei L, Liu J, Zhang J, Li J, Duan J and Fan D: Protective effects of Sparstolonin B, a selective TLR2 and TLR4 antagonist, on mouse endotoxin shock. Cytokine 75: 302-309, 2015 .

27. Liu Q, Wang J, Liang Q, Wang D, Luo Y, Li J, Janicki JS and Fan D: Sparstolonin B attenuates hypoxia-reoxygenation-induced cardiomyocyte inflammation. Exp Biol Med (Maywood) 239: 376-384, 2014

28. Cao Y, Guan K, He X, Wei CW, Zheng ZR, Zhang YH, Ma SL, Zhong $\mathrm{H}$ and Shi W: Yersinia YopJ negatively regulates IRF3-mediated antibacterial response through disruption of STING-mediated cytosolic DNA signaling. Biochim Biophys Acta 1863: 3148-3159, 2016. 